\title{
A Framework for Semi-Automatic Fiducial Localization in Volumetric Images
}

\author{
Dénes Ákos Nagy ${ }^{1}$, Tamás Haidegger ${ }^{2}$, and Ziv Yaniv ${ }^{1}$ \\ 1 The Sheikh Zayed Institute for Pediatric Surgical Innovation, Children's National \\ Health System, Washington, DC 20010, USA, \\ 2 Antal Bejczy Center for Intelligent Robotics, Obuda University, Hungary \\ ZYaniv@childrensnational.org
}

\begin{abstract}
Fiducial localization in volumetric images is a common task performed by image-guided navigation and augmented reality systems. These systems often rely on fiducials for image-space to physical-space registration, or as easily identifiable structures for registration validation purposes. Automated methods for fiducial localization in volumetric images are available. Unfortunately, these methods are not generalizable as they explicitly utilize strong a priori knowledge such as fiducial intensity values in $\mathrm{CT}$, or known spatial configurations as part of the algorithm. Thus, manual localization has remained the most general approach, readily applicable across fiducial types and imaging modalities. The main drawbacks of manual localization are the variability and accuracy errors associated with visual localization. We describe a semi-automatic fiducial localization approach that combines the strengths of the human operator and an underlying computational system. The operator identifies the rough location of the fiducial, and the computational system accurately localizes it via intensity based registration using the mutual information similarity measure. This approach is generic, implicitly accommodating for all fiducial types and imaging modalities. The framework was evaluated using five fiducial types and three imaging modalities. We obtained a maximal localization accuracy error of $0.35 \mathrm{~mm}$, with a maximal precision variability of $0.5 \mathrm{~mm}$.
\end{abstract}

\section{Introduction}

Surgical augmented reality and image-guided navigation systems aim to enhance the clinician's ability to interpret the underlying surgical scene observed via intra-operative imaging. This is most often achieved by registering high quality pre-operative images to the intra-operative physical setting [?,?]. Registration is typically performed using a rigid paired-point approach as it has an analytic solution [?]. Fiducials are localized in the pre-operative image and intra-operatively localized using a tracked calibrated pointer.

Performing paired point, rigid or non-rigid, registration in a robust, accurate, and precise manner is a key task in navigation and guidance systems, aligning image-space and physical-space. It is also in wide spread use in research laboratories where it serves as a means for estimating a ground truth transformation 
for evaluating results obtained with novel image-to-image or image-to-physical registration methods $[?, ?, ?]$.

The nomenclature describing the various errors in paired point registration was introduced in [?], defining the three relevant quantities: Fiducial Localization Error (FLE), Fiducial Registration Error (FRE), and Target Registration Error (TRE). Thus, to minimize the TRE we need to reduce FLE. In other words, we want to precisely and accurately localize the fiducials in image-space.

We next present, in chronological order, existing approaches to fiducial localization described in the literature.

The simplest form of localization is for a human operator to visually localize the fiducials in the volumetric image. This approach is common due to its simplicity and the robustness of the human operator to variations in imaging modalities and fiducial geometries and appearances. Studies that report the values of image-space FLE that can be expected in the clinical setting using skin adhesive fiducials were described in [?,?]. According to these reports, typical values for FLE in the image domain are in the range of $0.8-2.3 \mathrm{~mm}$. These depend upon the imaging modality, MR or $\mathrm{CT}$, associated spatial resolution, and the fiducial type in use. A study designed to evaluate FLE using a custom phantom with divot fiducials and manual localization in CT reported FLE values in the range of $0.4-0.8 \mathrm{~mm}$ [?]. Most likely these lower values are associated with the use of $\mathrm{CT}$ and the specific fiducial choice.

Possibly the first automated method was presented in [?]. Cylindrical markers with known dimension are automatically localized in CT and MR. The algorithm is comprised of two parts identification of potential fiducial locations and fiducial localization. The first part identifies potential fiducial locations using thresholding and morphological operations. The second part determines if a candidate is indeed a fiducial by comparing the size and shape of the connected component and the known fiducial size and radius. The fiducial is localized using the intensity weighted centroid of the connected component. It should be noted that in practice MR and CT are treated differently, with modality specific steps described as part of the algorithm. The method was shown to have a $1.4 \%$ false positive identification error rate localizing 168 fiducials.

In [?] an automated algorithm for localizing fiducials in CT and MR is presented. The method first identifies potential fiducial locations using the mathematical morphology top-hat operator; then, assuming no false negatives, it removes candidates that are too close to each other based on the intensity values in the candidate regions. Finally, localization is performed using the intensity weighted centroid of the region. The method was only evaluated on a single CT scan of a phantom with eight cylindrical fiducials. All fiducials were localized, but given the sample size it is unclear if this method can indeed deal with various fiducial types and modalities.

In [?] donut shaped fiducials are automatically localized in CT. A fixed intensity threshold is used to identify the patient and fiducials, this is followed by morphological operations that result in identification of connected components that are deemed to be fiducial locations. Each fiducial is localized using the in- 
tensity weighted centroid of the connected component. The method was shown to have less than $5 \%$ false negative and $2.3 \%$ false positive identification error rate localizing 25 fiducials.

In [?] a method based on template matching on the edge detection results of $\mathrm{CT}$ or MR is presented. The method is customized for donut fiducials that are commonly used by clinical systems. The method successfully identified fiducials in two CT and five MR data sets. Evaluation of the localization accuracy is not provided.

A method designed for automatic donut fiducial detection on cranial images is described [?]. The method is based on identification of fiducial corner points in the 2D edge detected result obtained from CT or MR. The corners are clustered via $\mathrm{K}$-means and a polynomial curve is fit to the corner points. The center point of the curves is defined as the fiducial location. The method successfully localized fiducials in 56 CTs with a difference of $0.5 \mathrm{~mm}$ or less from a manually defined ground truth. On $66 \mathrm{MR}$ images the method only failed twice.

Another method designed for automatic donut fiducial detection in cranial images is described in [?]. This method takes the 3D nature of the data into account and uses a local 2D height map based template matching approach to identify fiducial locations. First, the head surface is segmented from the CT or MR volume using a threshold. Then at each surface voxel location the distance between the surface and the plane defined by the voxel and the vector connecting it to the center of the volume/head is estimated. This local height map is then compared to the fiducial's height map. If the difference between the two height maps is less than a threshold this is a potential fiducial. All potential fiducial locations are clustered using a nearest neighbor approach. The marker is then localized by taking the candidate location whose height map is most similar to the fiducial's height map. The method was evaluated using $15 \mathrm{CT}$, and $10 \mathrm{MRI}$ data sets. In CT(MR) 69/75(47/52) fiducials were accurately localized.

A more recent method for automatic spherical fiducial localization in cranial $\mathrm{CT}$ images is described in [?]. The method is specific to $\mathrm{CT}$ as the intensity value of the fiducials is used as part of the localization approach. A set of surfaces is obtained from the CT using the marching cubes algorithm. The surface that corresponds to the head is removed and the remaining surfaces are categorized as fiducial or not based on the known fiducial geometry by comparing the surface's bounding box size to the expected size and the Hausdorff distance between the surface and a sphere positioned at the center of the bounding box. Once a surface is classified as a fiducial its location is estimated as the centroid of the surface vertices. The method was evaluated on clinical data with $211 / 233$ fiducials accurately localized.

It should be noted that each of these localization methods was evaluated using a single fiducial type, with some of the algorithms customized to the specific fiducial geometry or the anatomical structure on which they are attached, such as the head. In all of these algorithms the fiducial configuration is arbitrary. This necessitates visual confirmation as none of these algorithms can guarantee success, all fiducials correctly localized without any false positives. A strong 
constraint, that allows one to dispense with visual confirmation, is the use of a known fiducial configuration.

We propose to use a common framework for semi-automatic fiducial localization that is not tailored to a specific fiducial. The approach combines the strengths of a human operator and an underlying computational system. The operator performs a recognition task, identifying the presence of a fiducial in a rough location, and the computational system accurately localizes it. We next describe our approach in detail.

\section{Materials and Methods}

Our framework for fiducial localization in volumetric images is a natural extension of manual localization and thus fits within the existing clinical practice. We formulate fiducial localization as a multi-modality intensity based rigid registration task. Fiducials are modeled as volumetric images. In our case we use binary volumes, although other intensity models are readily accommodated by our framework. A straightforward method for creating additional fiducial models is to acquire a high quality volumetric scan of the new fiducial and accurately localize it in that image.

To localize a fiducial in an image the operator identifies the rough fiducial location in the image by clicking anywhere inside it, we then register the fiducial model to the image with the indicated location serving as the initial translational part of a rigid transformation. As most often fiducials represent a single point, we require that the origin of the model fiducial image be located at this point. Thus, the translational part of the transformation obtained by intensity based registration becomes the point we seek.

The fiducial model is registered locally, with the region of interest defined to be twice the size of the fiducial model image diameter. This ensures that even if the operator identified the fiducial's edge the whole fiducial is contained in our region of interest. The rigid transformation is parameterized using three translation components, with rotation represented by the three Euler angles. We use the Nelder-Mead downhill simplex as our optimizer, as it does not require the computation of derivatives. Given that our goal is to localize the fiducials in all modalities using the same framework, we use mutual information as our similarity measure [?,?].

As is well known, the success of iterative registration algorithms is highly dependent on initialization. In our case initialization only provides a constraint on the location of the fiducial and not its orientation. This is a critical issue, as registration will most likely fail if a correct registration means that the fiducial model image should be rotated by $180^{\circ}$ around one of its axes, which is not uncommon. We therefor associate a set of rotations with each fiducial model. Together with the location these define a set of initializations used to localize the fiducial. It should be noted that this set of orientations is dependent on the fiducial geometry. In case of a spherical fiducial there is only one rotation, the identity. Other commonly used fiducials such as the Beekly PinPoint (Beekley 

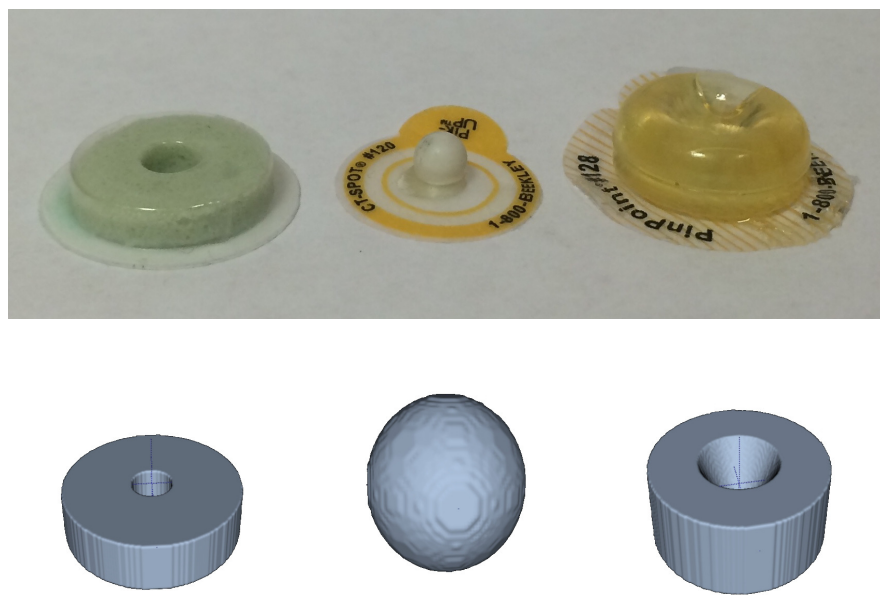

Fig. 1. Fiducial types used for framework evaluation: (top) physical fiducials (bottom) surface representation of the binary fiducial models.

Corp.,CT, USA) or the IZI multi modality markers (IZI Medical Products, MD, USA), shown in Figure ??, do require multiple orientations. As these two fiducial types are symmetric around one axis, they each have six orientations associated with them. We thus perform multiple registrations starting from the set of initializations defined by the fiducial geometry. The transformation obtained by the registration with the optimal similarity measure value is taken as the correct one.

An interesting issue with the IZI multi-modality markers is that they have two sets of symmetry planes. The more problematic one is due to the fact that the part that is adhered to the patient is symmetric to the top of this cylindrical fiducial. If we only modeled the fiducial this symmetry cannot be resolved. We therefor break the symmetry by slightly enlarging the image model so that it incorporates the fiducial, and above the fiducial an empty region. This matches the physical setup where the region above the fiducial contains air.

Our framework uses the following parameter values. For Mutual Information estimation we use 24 histogram bins and 0.4 of the pixels of the fiducial image. We set the function convergence tolerance to 0.001 , the parameters convergence tolerance to 0.25 and the maximum number of iterations to 200.

\section{Experimental Evaluation}

To evaluate our fiducial localization framework we used the following set of fiducials: (1) 4mm diameter sphere; (2) 6mm diameter sphere; (3) multi modality markers from IZI, referred to as donut fiducials; (4) the PinPoint multi modality markers from Beekly, referred to as cone fiducials; and (5) conical divot markers. 


\begin{tabular}{|c|c|c|c|c|c|}
\hline modality & $\begin{array}{c}\text { sphere } \\
4 \mathrm{~mm}\end{array}$ & $\begin{array}{l}\text { sphere } \\
6 \mathrm{~mm}\end{array}$ & donut & cone & divot \\
\hline $\mathrm{CT}$ & $5(1)$ & - & $5(1)$ & $5(1)$ & $12(1)$ \\
$\mathrm{CBCT}$ & $13(3)$ & $20(4)$ & - & - & - \\
$\mathrm{MR}$ & - & - & - & $5(1)$ & - \\
\hline \hline $\mathrm{CT}$ & - & - & $9(1)$ & - & - \\
$\mathrm{MR}$ & - & - & $15(2)$ & $9(1)$ & - \\
\hline
\end{tabular}

Table 1. Number of localized fiducials per modality, number of scans in parenthesis. Top part of the table are phantom data sets, bottom part are clinical data sets. Five different forms of fiducials were localized, for a total of 95 unique localizations.

Figure ?? shows a subset of these and corresponding surface models. The fiducials were imaged using three modalities: $\mathrm{CT}, \mathrm{MR}$ and Cone-Beam $\mathrm{CT}$ (CBCT). In some cases the images are of phantoms and in others clinical images. The data used in this evaluation study is described in Table 1.

The framework's precision was evaluated exhaustively. All of the fiducials are roughly localized in all data sets. We then perform an initial localization using the operator provided location as described above. The output of this localization serves as the input for our precision evaluation. We construct a set of concentric spheres centered on this point with each sphere having a radius $0.5 \mathrm{~mm}$ larger than the previous one. We randomly selected points on each of the spheres serving as user input for our framework. We quantify precision as the distance between the initial point used to construct the set of spheres and the result of each of the registrations.

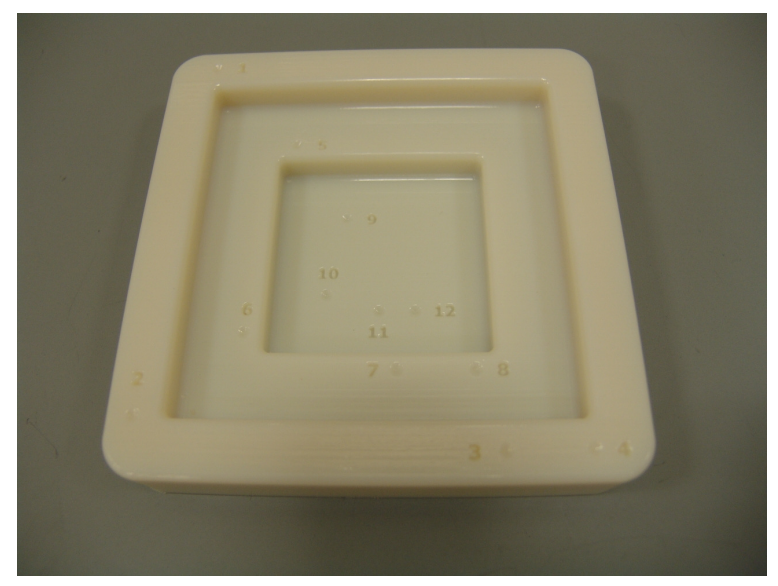

Fig. 2. Plastic phantom with divots whose location is known with high accuracy.

Evaluating accuracy is a non-trivial task, as the true fiducial location is never known. Instead we use common surrogates. In our case, we localized all spheri- 
cal fiducials using the weighted intensity centroid (implementation found online, ground truth for fiducial localization in CBCT [?]). We take the result of this approach as the reference fiducial location. In addition, we also designed a phantom with a dozen divot holes at known locations and used a highly accurate 3D printer, Objet500 Connex from Stratasys, to print it. Figure ?? shows this phantom. Finally, on two clinical data sets we used a common strategy for obtaining a reference localization; we had multiple, in our case five, operators manually localize donut fiducials and used the mean of this localization as the reference fiducial location.
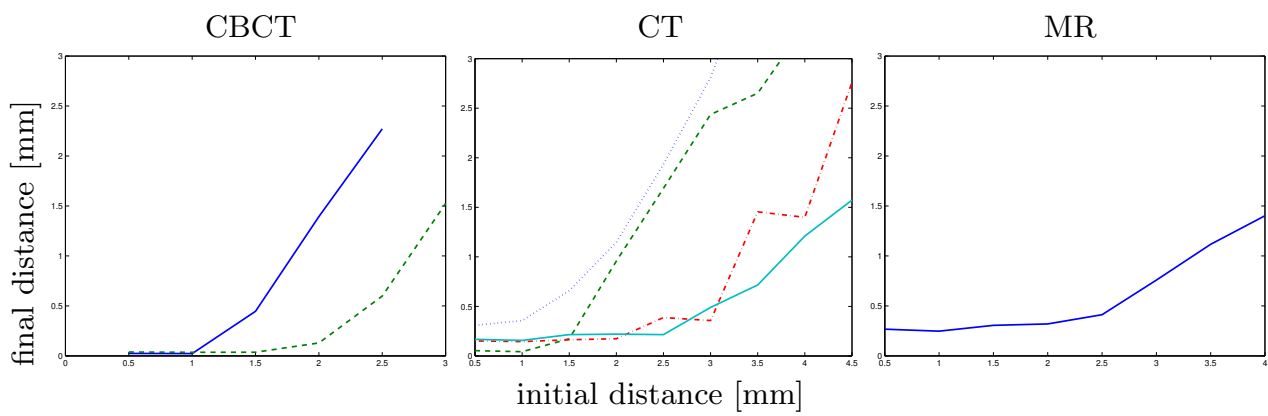

Fig. 3. Precision using phantom data. Each point on the graphs represents the average of 100 initializations. In CBCT we used $4 \mathrm{~mm}$ diameter spheres (continuous, blue, line) and $6 \mathrm{~mm}$ diameter spheres (dashed, green, line). In CT we used $4 \mathrm{~mm}$ spheres (dashed, green, line), cones (continuous, cyan, line), divots (dotted, blue, line) and donuts (dashdot, red, line). In MR we used cones.

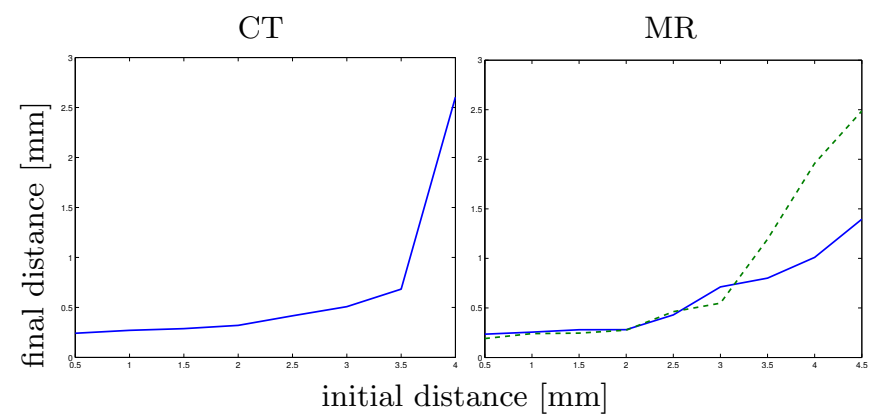

Fig. 4. Precision using clinical data. Each point on the graphs represents the average of 100 initializations. In CT we used donut fiducials. In MR we used cone fiducials (continuous, blue, line) and donut (dashed, green, line). 


\section{Results}

Our evaluation results for precision, both for phantom and clinical data, show a precision of less than $0.5 \mathrm{~mm}$ for all fiducial types. The breakdown point in precision occurs once the initialization is far enough from the actual fiducial location so that the whole fiducial is not encompassed by our region of interest. As the region of interest is determined by the fiducial size we observed that the breakdown point for larger fiducials was further away from their actual location, as is expected. Figures ?? and ?? summarize our experiments for phantom and clinical data.

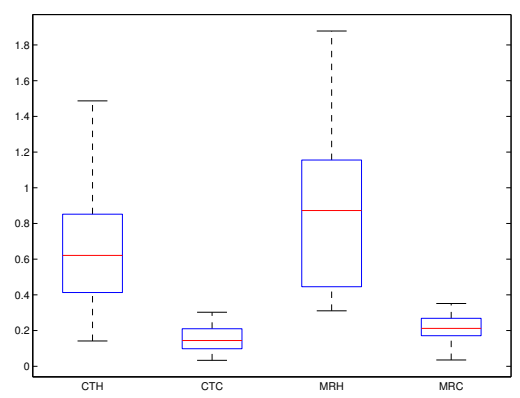

Fig. 5. Precision of fiducial localization in $\mathrm{mm}$. Donut fiducials localized in clinical CT and MR (CTH/MRH - manual, CTC/MRC - semi-automated). The precision of the semi-automated method is clearly better.

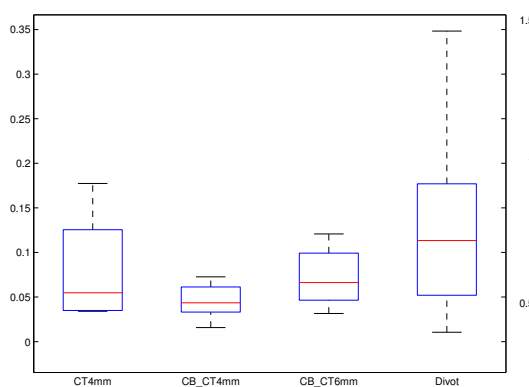

(a)

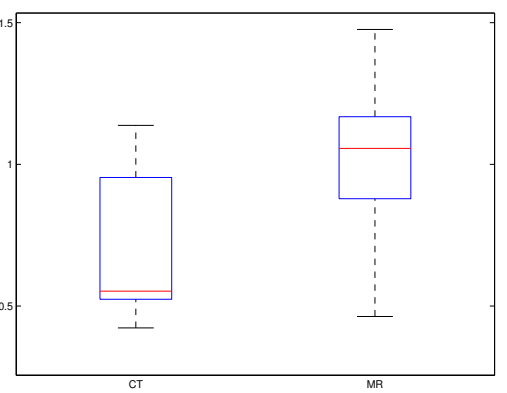

(b)

Fig. 6. Accuracy evaluation results in mm, (a) using phantom data and (b) using donut fiducials on clinical data.

While our results show that the proposed approach is highly precise, it is interesting to compare this approach to the human operator's precision. To compare the two, we took the manual "accurate" localizations used to define 
a ground truth on the clinical data. This illustrates the inter-observer variability and associated precision. We compare this to the variability associated with the proposed approach when the input is a rough localization. Figure ?? illustrates the higher precision of our method.

Having established that our framework is precise, we now look at its accuracy. Our framework obtained results with a maximal error of $0.35 \mathrm{~mm}$ on phantom data. This is in comparison to the ground truth obtained by using another semi-automated localization approach, intensity weighted centroid, and to a ground truth known from phantom construction. On clinical data our framework obtained results with a maximal error of $1.5 \mathrm{~mm}$. Figure ?? summarizes our accuracy evaluation. The accuracy results on the clinical data are significantly lower. Most likely this is due to the quality of our reference gold standard, averaging of multiple manual localizations. This observation is supported by the low precision exhibited by manual localization as seen in Figure ??

\section{$5 \quad$ Discussion and Conclusions}

We have presented a semi-automated framework for fiducial localization. The framework was evaluated for precision and accuracy using three fiducial geometries, sphere, cone, and donut. In all data sets all of the fiducials were accurately localized. Our approach is based on the robustness provided by the human operator, readily identifying the presence and rough location of fiducials. Once a fiducial is identified the registration based approach accurately and precisely localizes it.

While our approach is only semi-automatic, it is clinically acceptable as it is a slight modification of the existing workflow, and places less requirements on the operator. That is, instead of accurate localization the operator need only identify the rough location of the fiducial. In addition, by taking advantage

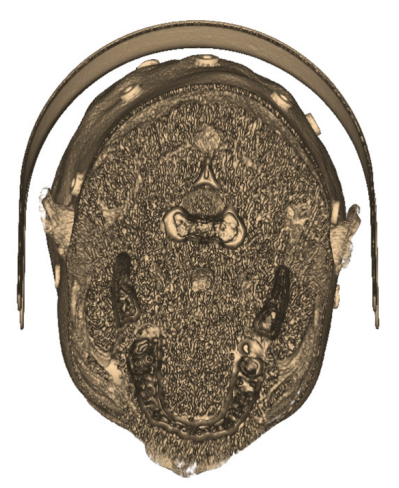

Fig. 7. Volume rendering of cranial CT image used in our evaluation. Note the presence of the head rest 
of the robustness of the operator's visual system complex settings are readily accommodated for in an implicit manner, something that is not trivial when proposing fully automated localization schemes. Point in case, we used a clinical CT for evaluation in which the patient's head was in a head rest (see Figure ??). None of the human operators was distracted by this, implicitly dealing with confounding information which would most likely cause automated methods to fail.

\section{Acknowledgment}

We would like to thank Kendall O'Brien for his help with image acquisition, and the following people for their help with the experiments Özgür Güler, Rahul Khare, Reza Monfaredi, Reza Seifabadi, and Emmanuel Wilson.

D. Nagy was supported by a grant from the Hungarian-American Enterprise Scholarship Fund.

\section{References}

1. Chen, D., Tan, J., Chaudhary, V., Sethi, I.K.: Automatic fiducial localization in brain images. International Journal of Computer Assisted Radiology and Surgery 1(1 Supplement), 45-47 (2006)

2. Cleary, K., Peters, T.M.: Image-guided interventions: technology review and clinical applications. Annu. Rev. Biomed. Eng. 12, 119-142 (2010)

3. Dang, H., Otake, Y., Schafer, S., Stayman, J.W., Kleinszig, G., Siewerdsen, J.H.: Robust methods for automatic image-to-world registration in cone-beam CT interventional guidance. Med. Phys. 39(10), 6484-6498 (2012)

4. Fallavollita, P., Aghaloo, Z.K., Burdette, E.C., Song, D.Y., Abolmaesumi, P., Fichtinger, G.: Registration between ultrasound and fluoroscopy or CT in prostate brachytherapy. Med Phys. 37(6), 2749-2760 (2010)

5. Gu, L., Peters, T.M.: 3D automatic fiducial marker localization approach for frameless stereotactic neuro-surgery navigation. In: Workshop on Medical Imaging and Augmented Reality (MIAR). pp. 329-336 (2004)

6. Horn, B.K.P.: Closed-form solution of absolute orientation using unit quaternions. Journal of the Optical Society of America A 4(4), 629-642 (April 1987)

7. Ji, S., Roberts, D.W., Hartov, A., Paulsen, K.D.: Intraoperative patient registration using volumetric true 3D ultrasound without fiducials. Med. Phys. 39(12), 7540$7552(2012)$

8. Liu, W., Ding, H., Han, H., Xue, Q., Sun, Z., Wang, G.: The study of fiducial localization error of image in point-based registration. In: International Conference of the IEEE Engineering in Medicine and Biology Society (EMBC). pp. 5088-5091 (2009)

9. Mattes, D., Haynor, D.R., Vesselle, H., Lewellen, T.K., Eubank, W.: PET-CT image registration in the chest using free-form deformations. IEEE Trans. Med. Imag. 22(1), 120-128 (2003)

10. Maurer, Jr., C.R., Fitzpatrick, J.M., Wang, M.Y., Galloway, Jr., R.L., Maciunas, R.J., Allen, G.S.: Registration of head volume images using implantable fiducial markers. IEEE Trans. Med. Imag. 16(4), 447-462 (1997) 
11. Nicolau, S., Garcia, A., Pennec, X., Soler, L., Ayache, N.: An augmented reality system to guide radio-frequency tumour ablation. Comput. Animat. Virtual Worlds 16(1), 1-10 (2005)

12. Shamir, R.R., Joskowicz, L., Spektor, S., Shoshan, Y.: Localization and registration accuracy in image guided neurosurgery: a clinical study. International Journal of Computer Assisted Radiology and Surgery 4(1), 45-52 (2009)

13. Tan, J., Chen, D., Chaudhary, V., Sethi, I.: A template based technique for automatic detection of fiducial markers in $3 \mathrm{D}$ brain images. International Journal of Computer Assisted Radiology and Surgery 1, 47-49 (2006)

14. Viola, P., III, W.M.W.: Alignment by maximization of mutual information. International Journal of Computer Vision 24(2), 137-154 (1997)

15. Wang, M., Song, Z.: Automatic localization of the center of fiducial markers in 3D CT/MRI images for image-guided neurosurgery. Pattern Recognition Letters 30(4), 414-420 (2009)

16. Wang, M.Y., Maurer, Jr., C.R., Fitzpatrick, J.M., Maciunas, R.J.: An automatic technique for finding and localizing externally attached markers in CT and MR volume images of the head. IEEE Trans. Biomed. Eng. 43(6), 627-637 (1996)

17. Woerdeman, P.A., Willems, P.W., Noordmans, H.J., van der Sprenke, J.W.B.: The effect of repetitive manual fiducial localization on target localization in image space. Neurosurgery 60(2 Suppl 1), ONS-100-ONS-103 (2007)

18. Yaniv, Z.: Localizing spherical fiducials in c-arm based cone-beam CT. Med. Phys. 36(11), 4957-4966 (2009)

19. Yaniv, Z., Cleary, K.: Image-guided procedures: A review. Tech. Rep. CAIMR TR2006-3, Image Science and Information Systems Center, Georgetown University (April 2006) 